\title{
Influence of the grafting topology of hydrophobic silica surfaces on the mechanical properties of silicone high consistency rubbers
}

\author{
David Mariot, ${ }^{a}$ Anne-Sophie Caro-Bretelle, ${ }^{\text {,** }}$ Patrick lenny ${ }^{\mathbf{b}}$ and François \\ Ganachaudc
}

\begin{abstract}
Silica-filled rubber materials exhibit stress softening and hysteresis under cyclic loadings. These phenomena are usually associated with both the Payne and Mullins effects. To better characterize these properties, five model silicas were produced and used in industrial-like high consistency rubber (HCR) formulations: the native (hydrophilic) silica and four chemically modified silicas for which both the content of surface silanols and the nature of the grafted silicone chains differ. Silica - polydimethylsiloxane HCR elastomer with constant silica content and optimal dispersion was tested via static (uniaxial tensile tests, cyclic and monotonic) and dynamic tests. The Payne and Mullins effects as well as the ultimate properties were evaluated as a function of particle surface treatment. It was found that the Payne amplitude decreases with the content of grafted chains, whereas the Mullins effect and ultimate strain decrease with an increase in molar mass between crosslinks. Finally, the ultimate strength is optimal as long as silica is surface treated, albeit independent of surface grafting topology.
\end{abstract}

Keywords: elastomers; coating; mechanical testing; mechanical properties

\section{INTRODUCTION}

Nano-reinforcement of rubbers is widely performed to enhance the mechanical properties of these materials, such as tensile stress and tensile strength, elongation at break, tear resistance etc. In particular, silicone materials are usually reinforced by nanoparticles of silica or carbon black, ${ }^{1}$ which offer a high specific surface area. The reinforcement of polydimethylsiloxane (PDMS) based elastomers is governed by particle - particle (aggregate structure) or particle-polymer interactions. In these composites, the polymer - particle bonds are clearly identified as hydrogen bonding between an $\mathrm{OH}$ group of the silica surface and an oxygen atom of the skeleton of the silicone chain. The particle - particle bonds (including hydrogen bonding) lead to an increase in the tensile strength (by a factor of 10$)^{2}$ until reaching an optimum fraction of filler incorporation (the so-called percolation threshold). It is well known that these connections lead to the formation of a layer of linked elastomer with a thickness between 1 and $5 \mathrm{~nm}$ which acts as an interphase between the filler and the matrix ${ }^{3}$ and which depends on the molar mass of the polymer chains. ${ }^{4}$ These interactions obviously determine the reinforcement of the final material. ${ }^{5}$ Unfortunately the strong interactions between the particles and the matrix lead to both a very poor level of dispersion and a high viscosity that is detrimental to the mechanical properties. To solve these problems, and also to avoid latent crepe hardening (see for example DeGroot and Macosko ${ }^{6}$ ), silica nanoparticles are functionalized (e.g. via organosilane grafting) before their incorporation in the matrix.

Under mechanical tests (monotonic and cyclic at low and large elongations), filled polymers exhibit nonlinear behaviour: after a few percent of deformation the stiffness drops (which appears simultaneously with a peak in viscosity). For higher deformations, unloading and loading produce an irreversible softening. These phenomena are commonly named the Mullins and the Payne effects. Although modelizations have been studied for a long time, $^{7,8}$ the link between phenomenology and microstructure is still a matter of discussion. The present report aims at studying these ill-understood mechanisms using real industrial materials, namely high consistency rubber (HCR) (to our knowledge, only one group has thoroughly studied the mechanical properties of a room temperature vulcanized silicone material). ${ }^{9}$ In addition, the mechanical properties (Payne, Mullins ultimate strain and strength) are derived as a function of silica surface treatment, where grafting density and topology have been precisely tailored as described below. The adopted procedure was (i) preparation and characterization of the nanofillers (pristine silica + four different surface treated silicas), (ii) preparation of the elastomers and characterization of their dispersion, (iii) evaluation of the mechanical properties and (iv) interpretation of the results using a simple damage model.

\footnotetext{
* Correspondence to: Anne-Sophie Caro-Bretelle, C2MA, Ecole des Mines d'Alès, 6 Avenue de Clavières, 30319 Alès, France.

E-mail: Anne-Sophie.Caro@mines-ales.fr

a Bluestar Silicones France S.A.S., 55 Avenue des Frères Perret, 69192 Saint-Fons Cedex, France

b C2MA, Ecole des Mines d'Alès, 6 Avenue de Clavières, 30319 Alès, France

c Ingénierie des Matériaux Polymères, UMR CNRS 5223, Bâtiment Jules Verne, 17
} avenue Jean Capelle, 69621 Villeurbanne, France 


\section{EXPERIMENTAL}

\section{Silica preparation and characterization}

A highly dispersible bare silica was first chosen, namely Aerosil ${ }^{\circledR} 200$ from Evonik Industries AG, Hanau-Wolfgang, Germany with a specific surface BET area of $200 \mathrm{~m}^{2} \mathrm{~g}^{-1}$ and a median diameter $d_{50}=12 \mathrm{~nm}$ (denoted as REF in the following). The surface modification steps consisted in grafting cyclosiloxanes either as is $\left(D_{4}\right.$ homopolymerization, treatments denoted $\left.t_{1}, t_{2}, t_{3}\right)$ or by introducing vinyl coupling groups $\left(D_{4} / D_{4}{ }^{V}\right.$ copolymerization, denoted $t_{4}$ ). The processes used for surface treatments are disclosed in a patent application ${ }^{10}$ and will not be detailed in this paper. The final grafting state of these silica surfaces, obtained from various characterization techniques [ ${ }^{29} \mathrm{Si}$ solid NMR, ethoxylation/gas chromatography (GC) technique], is given in Scheme 1 and their main features are summarized in Table 1. All treatments allowed (i) an easier filler dispersion in the matrix, (ii) a better control of surface - charge interaction by introducing a variety of grafting and surface silanol densities and (iii) the possibility of promoting chemical bonding with the surface coupling agent (for $t_{4}$ silica).

\section{Silicone material}

An HCR base supplied by Bluestar Silicones (Saint Fons, France) was systematically used in this study. The gum is a vinylterminated linear copolymer of dimethylsiloxane and methylvinylsiloxane $(0.075 \mathrm{~mol} \%)$, of average molar mass $M_{w}=500$ $000 \mathrm{~g} \mathrm{~mol}^{-1}$, mixed with a dihydroxy-terminated siloxane oil (used as a plasticizer) and silicas ( $45 \mathrm{phr}$ ) in a Z-arm shaped mill for $1 \mathrm{~h}$ ( $5 \mathrm{~h}$ for the REF silica). The peroxide curing agent (2,5-dimethyl2,5-di(tert-butylperoxy)hexane in a $50 \mathrm{wt} \%$ silicone paste from Akzo Nobel) was then added in a two-roll mill, and silicone sheets $\left(150 \times 150 \times 2 \mathrm{~mm}^{3}\right)$ were cured for $10 \mathrm{~min}$ at $170^{\circ} \mathrm{C}$ under 30 bars.

\section{Composite microstructure}

PDMS nanocomposite morphologies were studied using an optical microscope (Leika, Solms, Germany) with a 100 scale factor to study the macroscopic dispersion. The smallest visible mesh is composed of 9 pixels, which corresponds to a quantifying limit of silica aggregates of $12 \mu \mathrm{m}$ or more. A Quanta SEM 200 instrument (FEl, USA) with a 30000 scale factor was used for a more thorough microscopic analysis. Strips of material $2 \mathrm{~mm}$ thick were cryo-fractured and observed under an accelerating voltage of $5 \mathrm{kV}$. Image analyses (Aphelion ${ }^{\mathrm{TM}}$ 3.2, ADCIS, France) were done for each scale of observation using several micrographs (at least five) in order to cover the whole specimen surface area. Micrographs were first converted into black and white images; silica aggregates were then identified, numbered and filtered by their surface area values $(A)$ at each scale of observation.

\section{Molar mass between crosslinking points}

Swelling measurements were carried out to get an indirect estimation of the average chain length $M_{c}$ between two crosslinking points. These experiments were performed in methylcyclohexane to determine the overall crosslinking density. The mean molar mass can be calculated from the Flory-Rehner equation: ${ }^{11}$

$$
M_{c}=-\frac{M_{s o l} * \rho_{s i l} / \rho_{s o l} *\left(V_{r T}^{1 / 3}-V_{r T} / 2\right)}{\ln \left(1-V_{r T}\right)+V_{r T}+\chi * V_{r T}^{2}}
$$

where $\chi, M_{\text {sol }}, \rho_{\text {sol }}$ and $\rho_{\text {sil }}$ are the Flory-Huggins interaction parameter, the molar mass of solvent, and the solvent and silicone densities, respectively. $V_{\mathrm{rT}}$ expresses the polymer fraction in the swollen polymer that is composed of polymer, silica (not swollen) and solvent. The determination of $V_{\mathrm{rT}}$ is performed as follows. A disc of $13 \mathrm{~mm}$ diameter and $2 \mathrm{~mm}$ thickness was first weighed to determine the initial weight $\left(W_{\mathrm{i}}\right)$ and then immersed in $100 \mathrm{~mL}$ of cyclohexane in a sealed bottle. After $48 \mathrm{~h}$ at room temperature, the equilibrium was considered to be reached as soon as the swollen silicone disc showed a constant weight. After a quick surface drying with an absorbing paper to remove the solvent that did not participate in the swelling, the silicone disc was weighed a second time $\left(W_{s}\right)$. The swollen disc was then dried in a ventilated oven to obtain the final weight $\left(W_{\mathrm{f}}\right)$. This value $W_{\mathrm{f}}$ is strictly inferior to $W_{\mathrm{i}}$ since a small part of uncrosslinked PDMS is extracted during the swelling step. $V_{\mathrm{rT}}$ is deduced from the equation

$$
V_{r T}=\frac{W_{f}-\left(W_{i} * c\right)}{W_{f}-\left(W_{i} * c\right)+\left(W_{s}-W_{f}\right) * \frac{\rho_{s i l}}{\rho_{s o l v}}}
$$

where $c$ represents the silica weight fraction, which was taken to be constant in all these tests.

\section{Tensile, cyclic quasi-static and dynamic tests}

The linear and nonlinear viscoelastic behaviours were characterized at low strain (dynamic mechanical analysis) and large strain (cyclic and monotonic loadings). Dynamic mechanical analyses were carried out with a Metravib viscoanalyser which is a dynamic shear rheometer. The tests were conducted on cylindrical samples (diameter $25 \mathrm{~mm}$, height $1 \mathrm{~mm}$ ). Isothermal dynamic sweep measurements were performed at ambient temperature. The samples were sinusoidally stretched under various strain magnitudes at a frequency of $0.314 \mathrm{rad} \mathrm{s}^{-1}$. The stress-strain responses were recorded automatically and used for evaluation of the storage modulus $G^{\prime}$, the loss modulus $G^{\prime \prime}$ and $\tan \delta$ which is the ratio of $G^{\prime \prime}$ and $G^{\prime}$.

Uniaxial tensile tests were conducted on a Zwick proline Z005 testing machine according to the ASTM-D412 standard. Tested specimens are plane dogbones (H2 type: length $L=75 \mathrm{~mm}$, height $I=4 \mathrm{~mm}$, width $h=2 \mathrm{~mm}$ ). The crosshead speed was taken as $500 \mathrm{~mm} \mathrm{~min}^{-1}$ and the software TestXpert ${ }^{\circledR}$ allowed us to record time, load $(F)$ and elongation $(\Delta L)$ from which strain was calculated $(\varepsilon=\Delta L / L)$. The nominal stress is defined as $\sigma=F / l h$. The tensile tests were conducted up to rupture of the specimen (to characterize the ultimate properties tensile strength $\sigma_{\mathrm{u}}$, ultimate elongation $\varepsilon_{\mathrm{u}}$ ) and were achieved first with five loadings - unloadings up to a strain level of about $200 \%(\varepsilon=2)$ and then successively up to about $400 \%(\varepsilon=4)$ and $600 \%(\varepsilon=6)$ to specifically characterize the Mullins effect. All presented values are mean curves of at least five tensile test experiments.

\section{RESULTS AND DISCUSSION \\ Elastomer preparations and characterizations}

HCR elastomers were prepared using conventional industrial formulation and mixing techniques (see Experimental for details). Starting from the same bare silica, exhibiting a high surface area, we polymerized different cyclosiloxanes $\left(D_{4}\right.$ or a combination of $D_{4} / D_{4} v$ ) onto the silica surface to create different grafting topologies, schematized in Scheme 1. From both chemical 
(a)

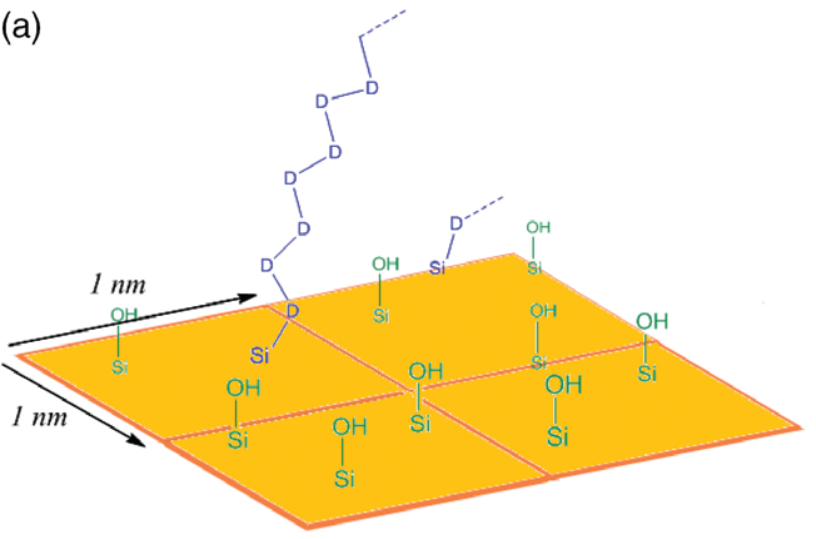

(c)

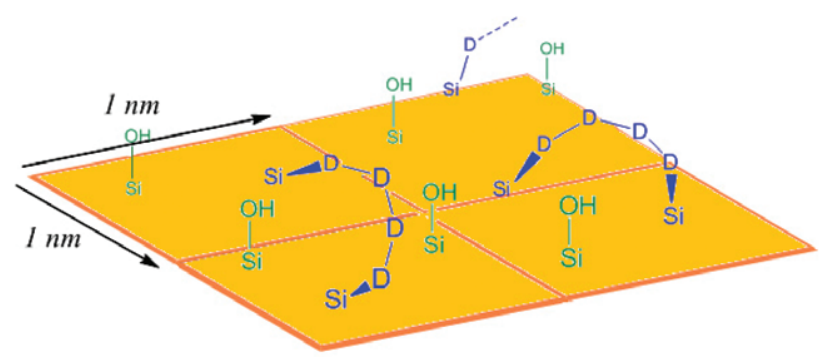

(b)

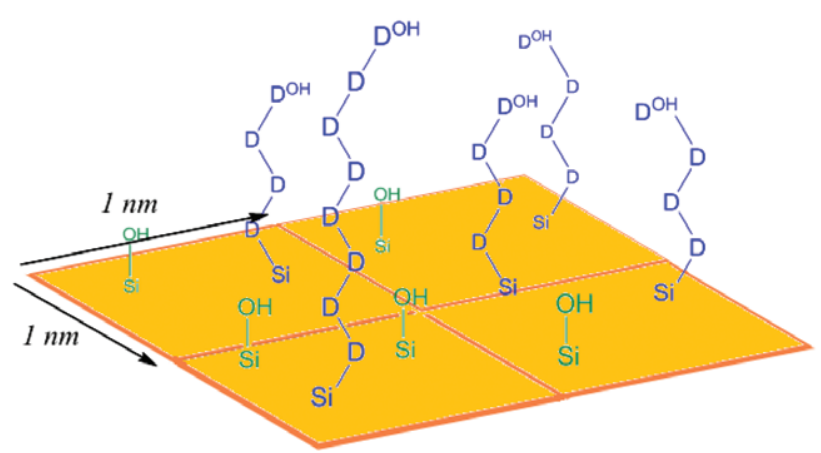

(d)

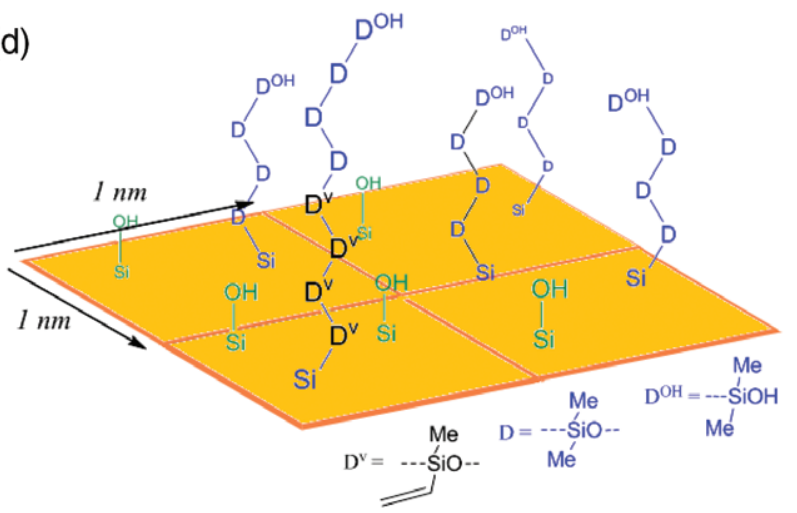

Scheme 1. Grafting topology of the various silicas used in this work (the reference, not shown, is fully hydroxylated): (a) $t_{1}$, 'long' chains of PDMS at low surface density; (b) $t_{2}$, short chains with large surface covering; (c) $t_{3}$, short silicone loops; (d) $t_{4}$, the same as $t_{2}$ but with $0.51 \%$ of vinyl groups in the chains (in the graph, much fewer vinyl groups are available on the surface: this schematic view is given to show that the grafted chains first incorporate $D^{V}$ units and then D units).

Table 1. Main features of silicas, swelling results and modelling of the mechanical properties

\begin{tabular}{|c|c|c|c|c|c|c|}
\hline & & REF & $t_{1}$ & $t_{2}$ & $t_{3}$ & $t_{4}$ \\
\hline \multirow[t]{3}{*}{ Silica properties } & $n_{\mathrm{SiOH}}{ }^{\mathrm{a}}\left(\mathrm{nm}^{-2}\right)$ & 4.60 & 3.60 & 2.50 & 2.65 & 2.25 \\
\hline & $n_{D}{ }^{b}\left(n^{-2}\right)$ & 0 & 3.80 & 3.10 & 3.00 & $3.65^{\mathrm{d}}$ \\
\hline & $\mathrm{DP}_{\text {graft }^{\mathrm{C}}}\left(\mathrm{g} \mathrm{mol}^{-1}\right)$ & 0 & 3.80 & 1.50 & 1.55 & 1.55 \\
\hline Swelling measurements & $M_{\mathrm{c}}\left(\mathrm{g} \mathrm{mol}^{-1}\right)$ & 9850 & 19850 & 18450 & 18650 & 8500 \\
\hline \multirow{2}{*}{ Mechanical properties at break } & $\varepsilon_{\mathrm{u}}(\%)$ & 500 & 880 & 820 & 850 & 460 \\
\hline & $\sigma_{\mathrm{u}}(\mathrm{MPa})$ & 9.4 & 10.0 & 10.5 & 10.7 & 10.2 \\
\hline \multirow[t]{2}{*}{ Model fitting } & $d_{\infty}$ & 0.66 & 0.45 & 0.52 & 0.52 & 0.68 \\
\hline & $\eta$ & 0.7 & 0.62 & 2.03 & 1.20 & 0.77 \\
\hline \multicolumn{7}{|c|}{$\begin{array}{l}\text { a Density of SiOH groups at the silica surface, obtained from }{ }^{29} \mathrm{Si} \text { solid NMR by integrating and comparing the } Q_{2}, Q_{3} \text { and } Q_{4} \text { areas. } \\
\text { b Density of grafted D units obtained by measuring the content of each unit (M, D, T and } Q \text { ) by a digestion technique and Gas Chromatography (GC) } \\
\text { analysis, and normalizing the } D \text { unit contents by each silica specific surface obtained by BET. } \\
{ }^{c} \text { Average graft degree of polymerization obtained from } n_{\mathrm{SiOH}} / n_{\mathrm{D}} \text {. } \\
\text { d Including } 0.17 \text { vinyl functions per square nanometre. }\end{array}$} \\
\hline
\end{tabular}

characterization techniques and swelling measurements, we were able to describe each surface treatment by the densities of surface silanol and siloxane units, from which average degrees of polymerization were deduced (see Table 1).

\section{Silica grafting topology}

As a reference, the surface of bare silica was first fully hydroxylated for 3 days in an acidic water solution heated at $60^{\circ} \mathrm{C}$. After drying, the ${ }^{29} \mathrm{Si}$ NMR spectrum of this silica was deconvoluted to extract a reference spectrum. We used here the surface silanol density of fully hydrated fumed silica at about $4.6 \mathrm{SiOH} \mathrm{nm}{ }^{-2}$ as detailed in the work of Zhuravlev. ${ }^{12}$ After treatment, the modified silicas were analysed under the same conditions to obtain the density of surface silanols $n_{\mathrm{SiOH}}$. As expected, the surface treatment impacts the number of surface silanols significantly, showing a global decrease in comparison with native silica. Note, however, that in the best case more than $60 \%$ silanol still remains accessible for physical interactions. The lengths of the grafted oligomers are on average quite short, even though some differences are traceable between silica $t_{1}$ and the others (see Table 1 ). 


\section{Crosslinking densities}

The results of swelling experiments performed on crosslinked elastomers are presented in Table 1. From these, we determine $M_{c}$ the mean molar mass between two crosslinking points, the latter including reversible knots (from silica/PDMS physical interactions) and irreversible chemical links. These $M_{c}$ values highlight the impact of the silica grafting content on the network density. Elastomers reinforced with D-modified silicas have a mean molar mass between crosslinking points of $18500-20000 \mathrm{~g} \mathrm{~mol}^{-1}$, independent of the silica surface grafting topology. The presence of vinyl grafted at the silica surface ( $t_{4}$ treatment) induces a decrease of $M_{c^{\prime}}$ indicating that these chemical groups react during the curing step and create chemical bonds between the silica filler and the silicone matrix. The low $M_{c}$ value found with the REF sample is ascribed to the hydrogen bonding that the silicone chains share with the silica surface through silanol functions.

\section{Silica dispersions}

Silica dispersion in the silicone material point is very important since a strong relation between microstructure and mechanical behaviour has already been observed..$^{13}$ For all the formulations, many of the elementary particles cluster together to form silica aggregates. The dispersion and distribution of the reference sample, initially bad, were optimized by mixing during a longer time prior to vulcanization (see Experimental). For the formulations making use of treated silicas, both scales of observation (optical microscopy and SEM) show that, whatever the functionalization, a good dispersion is obtained. No big agglomerates (particle size over $150 \mu \mathrm{m}$ ) were detected by optical microscopy. Small differences between elastomers could be observed, however, when looking at agglomerates of $50 \mu \mathrm{m}$ size, explaining the slight differences in the translucency of thin elastomer strips (Fig. 1).

Image analysis from SEM and optical micrographs enable a quantitative characterization of particle dispersion and distribution. By collecting data from each magnitude analysis, the percentage of particle diameters can be obtained from $d=0.1 \mu \mathrm{m}$ to $d=0.25 \mathrm{~mm}$ (the aggregates are assumed to be circular, $\left.d=(4 A / \pi)^{0.5}\right)$. At $\times 2 \mathrm{k}$ zoom, some white dots can be observed on almost all pictures, characteristic of slightly agglomerated particles. At $\times 50 \mathrm{k}$ zoom, no differences are revealed by the microscopy; the morphology of the dispersed silica seems to be the same, independently of the surface treatment. This is shown by the median diameters that are quite insensitive to surface treatment (between 0.015 and $0.02 \mathrm{~mm}$ for the macroscopic scale and under $0.15 \mu \mathrm{m}$ for the microscopic scale) (Fig. 1).

All these formulations are thus equivalent from a microstructural point of view. Deviation in the mechanical properties can therefore be correlated to the particle surface treatment. One referee suggested that we carry out SAXS or (nano)tomography to better argue on this point. The statement on the microstructure based on both experience of these materials and a careful optical microscopy image analysis was deemed sufficient in this industrially related study. (Note that the material filled with the REF silica, contaminated during the image analysis, is not presented in Fig. 1.)

\section{Mechanical properties}

\section{The Payne effect on non-vulcanized elastomers}

The Payne effect is observed under cyclic loading conditions at small deformations (under $100 \%$ strain when the matrix shows a linear mechanical behaviour); it is known, for the filled rubber, as the decrease in storage modulus (and a maximum in loss modulus) when the strain amplitude increases. This phenomenology is often associated with a breakdown process occurring in the agglomerates ${ }^{14}$ and was shown to be fully recoverable. ${ }^{9} \mathrm{Nev}$ ertheless some researchers have reported that this nonlinear effect strongly depends on the filler surface treatment. ${ }^{15-17}$ In fact the mechanisms responsible for the Payne effect still remain controversial.

Figure 2(a) shows the storage modulus versus strain amplitude for the different mixtures before vulcanization. First, these curves, independent of the surface treatment, reproduce the classical viscoelastic behaviour of filled polymer. The effect of surface treatment is also clearly demonstrated: a non-modified surface leads to higher storage modulus. Nevertheless the maximum loss modulus is reached for the same level of strain for all the formulations and approximately corresponds to the break point in the storage modulus. The Payne effect, evaluated by $G_{0}^{\prime}$ (the storage modulus for small strain amplitude), is found to vary with the silica surface silanol content (Fig. 2(b)): higher concentrations of silanol induce a larger Payne effect. This trend seems to confirm that filler surface treatment should be responsible for the Payne effect. ${ }^{18}$ In the case of filled organic rubber, some researchers made the same observation: the Payne effect is closely related to the amount of bound rubber; a linear evolution is observed when $\Delta G^{\prime}\left(G_{\infty}^{\prime}-G_{0}^{\prime}\right)$ is plotted versus the amount of bound rubber (grams per gram of silica). ${ }^{3}$ The dissipation of the deformation also seemed to depend strongly on $M_{\mathrm{c}}$ (not shown).

\section{Monotonic tensile tests}

Strain-stress tensile test curves of reinforced silicone elastomers filled with the different modified silicas show similar trends (Fig. 3). Their deformation is spatially uniform without any necking phenomena (as a consequence of a silica surface area lower than $\left.300 \mathrm{~m}^{2} \mathrm{~g}^{-1}\right){ }^{19}$ At low stress $(<50 \%)$, a small modulus drop is observed, followed by a slight modulus increase when increasing elongation (between 50\% and 300\%). Regarding ultimate properties (tensile strength and elongation at break), all tested reinforced elastomers have a tensile strength between 10 and $11 \mathrm{MPa}$ (within the error of the test, see Fig. 3(a)). Tensile strength is not a priori correlated to $M_{\mathrm{c}}$. It is widely reported in the literature that $\sigma_{\mathrm{u}}$ depends mainly on the specific surface area and microstructural state of the silica particles, independent of their surface chemistry. ${ }^{17}$ The constant value observed here reflects the maximum load that the chains, stuck heavily on the surface, can physically bear before they break. In contrast, ultimate elongation is clearly sensitive to surface treatment. In Fig. 3(b) we see that the ultimate strain $\varepsilon_{\mathrm{u}}$ improves when increasing $M_{c}$, at least in the range $M_{c} \in[8000$; $\left.20000 \mathrm{~g} \mathrm{~mol}^{-1}\right]$. Recent studies showed that above this threshold value of $20000 \mathrm{~g} \mathrm{~mol}^{-1}$ the material is weakened again because of the lower crosslinking density. ${ }^{20}$

\section{Cyclic tensile tests}

Typical cyclic nominal stress-strain curves are shown in Fig. 4 for all samples. They reveal the Mullins effect as well as the material dissipative effect as viscoelasticity. Each loading sequence (three strain levels: $200 \%, 400 \%$ and $600 \%$ ) is followed by unloading until $20 \%$ of the maximum load achieved during loading (such a shortcut allows some time to be saved in the lengthy tensile tests). In practice, this cyclic loading consists of five cycles for each given level of stretch. For the reference formulation an additional sequence is performed at a lower strain level (strain level 100\%) 

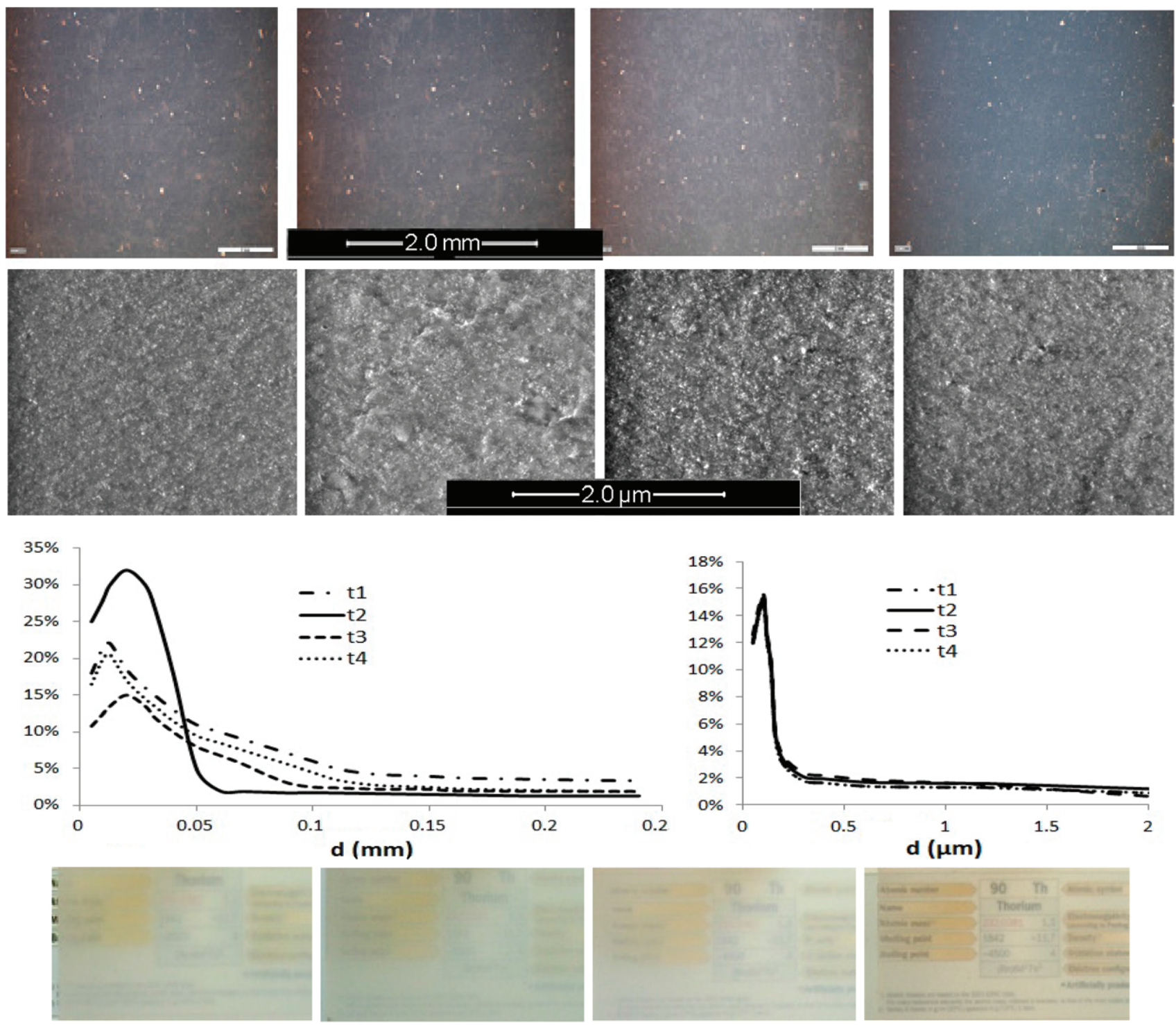

Figure 1. PDMS/treated-silica elastomers as imaged by optical microscopy (top row, the length bar is $2 \mathrm{~mm}$ ) and SEM (second row, the length bar is $2 \mu \mathrm{m}$ ). Elastomer cuts shown from left to right are filled with $t_{1}, t_{2}, t_{3}$ and $t_{4}$ silicas. Third row: aggregate size distribution as revealed by image analysis of optical (left) and SEM (right) micrographs (plotted as the content of particles versus their size, REF omitted). Last row: translucency of the different elastomers (the REF sample was more transparent but yellowy, not shown).

due to its poor tensile resistance. For all the formulations, loading and unloading paths following the first cycle do not rigorously coincide. The five cycles finally led to material stabilization, thus removing the viscoelastic response from the different materials.

The significant hysteresis observed during the first loading cycle compared to the fifth sequence increases strongly with the applied maximum deformation. The materials present a stress softening that depends quite strongly on surface treatment. This phenomenon is widely known as the Mullins effect ${ }^{3}$ and is associated with the breakage of links inside the material (filler - matrix links and chain interaction links). ${ }^{5,7,8}$ The Mullins effect is usually attributed to bond rupture, molecules slipping, filler rupture, disentanglement ${ }^{21-23}$ (including disentanglement density). ${ }^{24}$ Some researchers have reported a complete recovery of the stress softening at room temperature, usually observed after heating of solvent exposure. ${ }^{25}$

Some models combined damage, viscoelasticity and viscoplasticity of particle-reinforced rubbers ${ }^{26,27}$ to mechanically describe this effect. For easier interpretation of the microstructure/mechanics relationships, we chose to represent the damage of a material $(d)$ as a function of strain. The damage function can be estimated for each level of cyclic loading $(\varepsilon=2,4,6)$ by taking the ratio between the loading and unloading curve areas (see Fig. 5(a))

For each material, we have extrapolated the fifth unloading curve to zero strain and calculated the area under the curve accordingly. The error made by doing so was estimated by a different calculus method at less than 10\% (see the error bars in Fig. 5(a)). Note that for the REF sample $100 \%$ and $200 \%$ strains were used to calculate the two parameters, from which the values obtained compare well with other materials. The damage function can be fitted using a two-parameter model: ${ }^{7}$

$$
\begin{gathered}
d(\varepsilon)=d_{\infty}\{1-\exp [-(s+1) / \eta]\} \\
d, d_{\infty} \varepsilon[0,1] \quad \eta \geq 0
\end{gathered}
$$



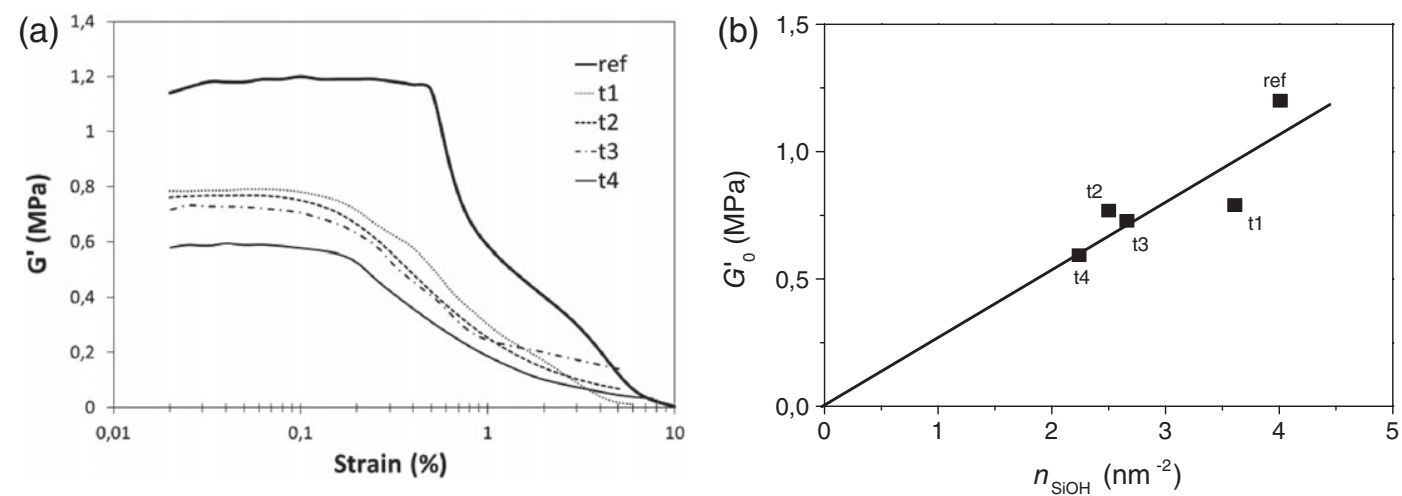

Figure 2. (a) Storage modulus versus strain amplitude of non-vulcanized formulations. (b) Initial storage modulus versus silanol density on treated silicas (line is just a guide for the eye).
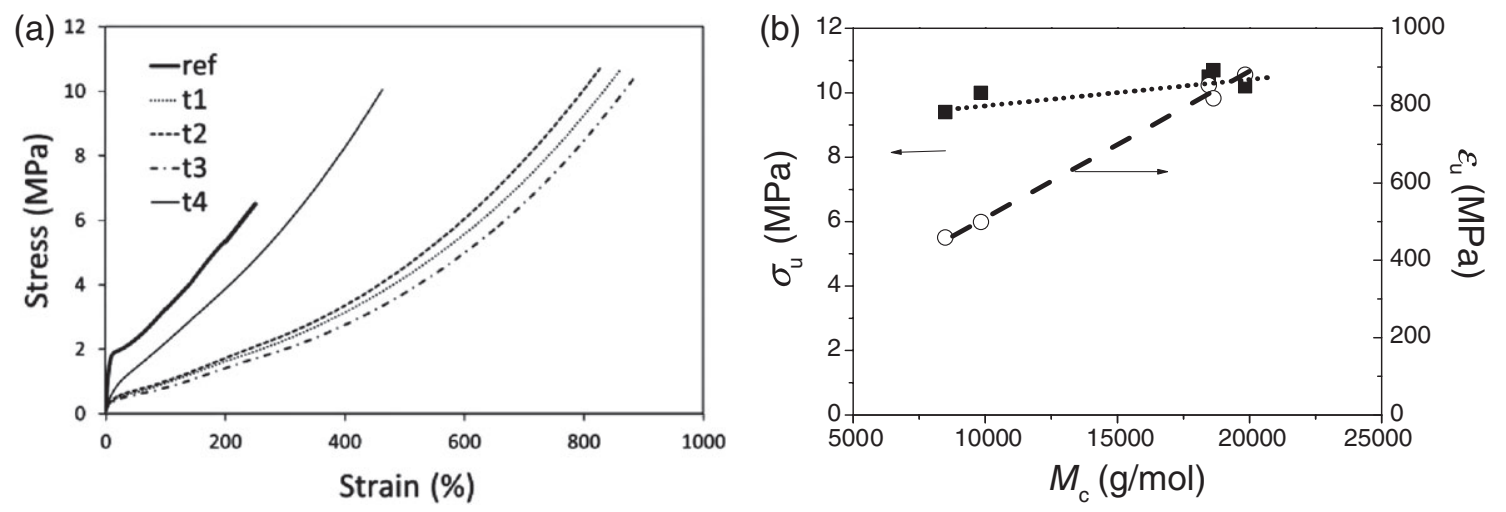

Figure 3. Tensile tests: (a) stress versus strain for the different elastomers; (b) evolution of ultimate mechanical properties against surface treatment.

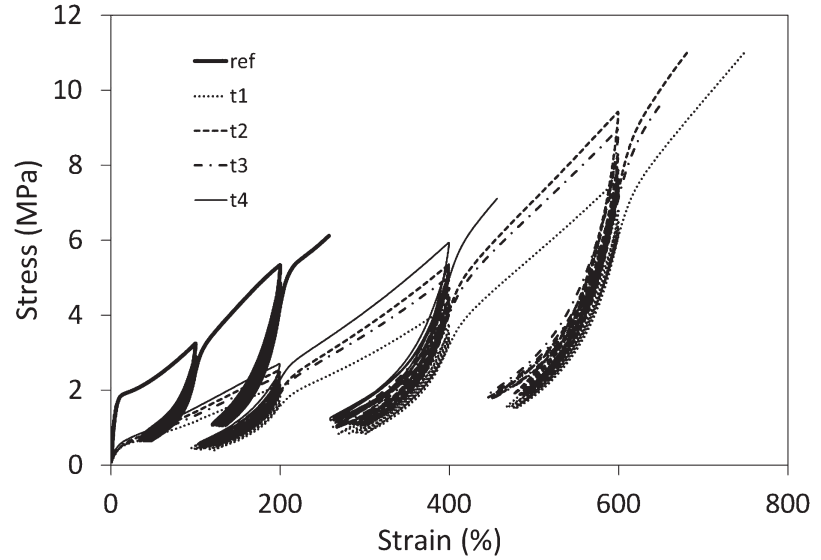

Figure 4. Cyclic loading tests showing the effect of functionalization on the Mullins effect for all materials.

with $d_{\infty}$ the global damage done to the material ( 1 means a fully damaged material) and $\eta$ the damage accumulation.

The two $d_{\infty}$ and $\eta$ parameters determined for each formulation are quoted in Table 1 (for details, a more thorough presentation of this procedure can be found in Caro-Bretelle et al. ${ }^{28}$ ). First, the silica covered with vinyl groups follows the trend of the reference (a highly reinforcing silica), whereas other silica-filled materials are less damaged. For all the surface treatments (including those bearing functional vinyl groups), the $d_{\infty}$ parameter decreases with $M_{c}$, meaning that a denser silica network stands the load applied to the test sample better. On the other hand, the parameter $\eta$ was found to decrease with increasing surface silanols except for the silica $t_{4}$ (Fig. $5(\mathrm{~b})$ ). This last result means that the Mullins effect cannot be explained by considering independently the content of physical crosslinking occurring with silica or the silicone network density. Such results agree with the theory involving both chain sliding (in the case of physical crosslinking) and chain breaking (chemical crosslinking).

\section{CONCLUSION}

The aim of this paper was to give some hints about the influence of silica's surface treatments on the mechanical properties of industrially viable HCR. Silicone elastomers were filled with a defined weight fraction of functionalized silica, produced by different processes and for which the grafting topology was perfectly mastered. Silica dispersions being comparable between samples, mechanical properties were found to be a direct consequence of particle surface treatment. Both non-functional and vinyl treated silicas were tested. Most parameters could be related to the crosslink density and/or the quantity of remaining silanols on the silica surface, bearing in mind that these two parameters are not independent.

The main conclusions can be summarized as follows:

(i) The Payne effect depends on silanol surface concentration and thus seems to be governed mainly by particle - polymer interactions.

(ii) The damage associated with the Mullins effect decreases with an increasing average chain length between crosslinking points and is thus related to the physical and chemical crosslink densities. 

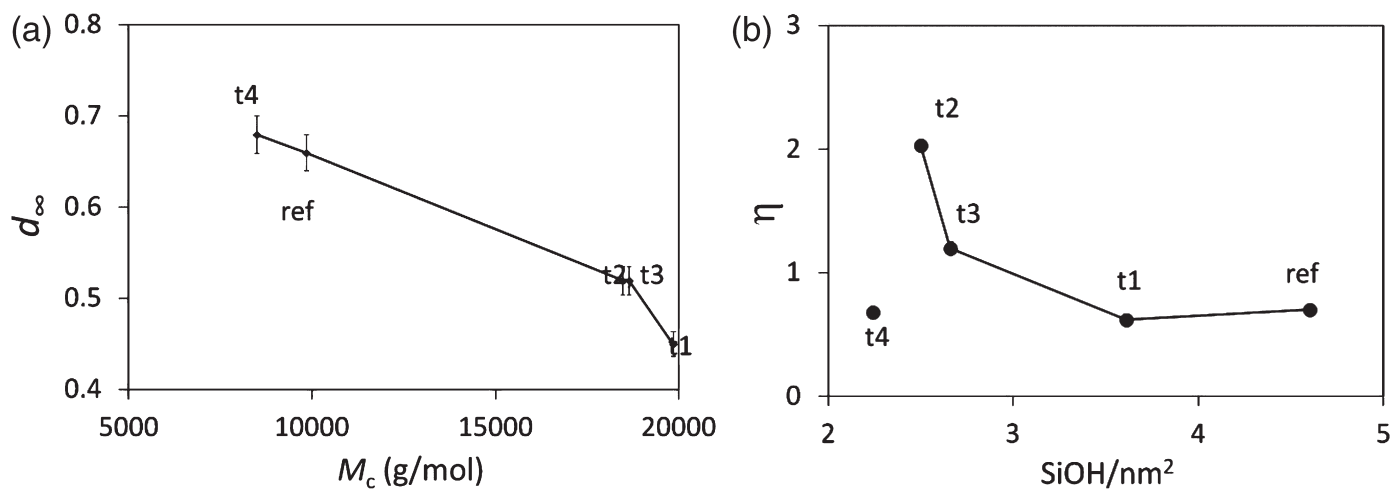

Figure 5. (a) Global damage versus $M_{c}$. (b) Damage accumulation versus silica treatment (as given by the remaining silanol contents on silica surfaces).

(iii) The ultimate strain increases with the average chain length between crosslinks, which is a consequence of the crosslinking density evolution.

\section{ACKNOWLEDGEMENTS}

We are grateful to Bluestar Silicones for providing all the materials that were necessary to carry out this study.

\section{REFERENCES}

1 Leblanc JL, Prog Polym Sci 27:627-687 (2002).

2 Polmanteer KE and Lentz CW, Rubber Chem Technol 48:795-809 (1975).

3 Ramier J, Gauthier C, Chazeau L, Stelandre L and Guy L, J Polym Sci B Polym Phys 45:286-298 (2007).

4 Mullins LJ, Rubber Chem Technol 21:281-300 (1948).

5 Bueche F, J Appl Polym Sci 5:271 -281 (1961).

6 DeGroot JV and Macosko CW, J Colloid Interface Sci 217:86-93 (1999).

7 Chagnon G, Verron E, Gornet L, Marckmann G and Charrier P, J Mech Phys Solids 52:1627-1650 (2004).

8 Chagnon G, Verron E, Marckmann G and Gornet L, Int J Solids Struct 43:6817-6831 (2006).

9 Chazeau L, Brown JD, Yanyo LC and Sternstein S, Polym Compos 21:202-222 (2000).

10 Mariot D and Ganachaud F, Method for preparing a silica grafted with an organosilicon compound. WO2013024137, France (2012).
11 Flory PJ and Rehner J, J Chem Phys 43:6817-6831 (1943).

12 Zhuravlev LT, Colloids Surf A Physicochem Eng Asp 173:1 - 38 (2000).

13 Bokobza L, Rubber Chem Technol 86:423-448 (2013).

14 Kraus G, J Appl Polym Sci Appl Polym Symp 39:75-92 (1984).

15 Maier PG and Gritz D, Kautschuk Gummi Kunststoffe 49:18-21 (1996).

16 Cassagnau P and Mélis F, Polymer 44:6607-6615 (2003).

17 Omnès $\mathrm{B}$, Thuillier S, Pilvin P, Grohens $\mathrm{Y}$ and Gillet $\mathrm{S}$, Compos Part A Appl Sci Manuf 39:1141-1149 (2008).

18 Paquien JN, Galy J, Gérard JF and Pouchelon A, Colloids Surf A Physicochem Eng Asp 260:165-172 (2005).

19 Ogebule O, Medvedev GA and Caruthers JM, Polymer 54:1190-1196 (2013).

20 Delebecq E, Hermeline N, Flers A and Ganachaud F, ACS Appl Mater Interfaces 4:3353-3363 (2012).

21 Diani J, Fayolle B and Gilormini P, Eur Polym J 45:601 -612 (2009).

22 Clément F, Bokobza L and Monnerie L, Rubber Chem Technol 78:211-231 (2005).

23 Clément $F$, Bokobza $L$ and Monnerie L, Rubber Chem Technol 78:232-244 (2005).

24 Hanson DE, Hawley M, Houlton R, Chitanvis K, Rae P, Orler BE et al, Polymer 46:10989-10995 (2005).

25 Yan L, Dillard DA, West RL, Lower LD and Gordon GV, J Polym Sci B Polym Phys 48:2207-2214 (2010).

26 De Tommasi D, Puglisi G and Saccomandi G, J Rheol 50:495-512 (2006).

27 Diani J, Brieu M and Vacherand JM, Eur J Mech A Solids 25:483-496 (2006).

28 Caro-Bretelle AS, lenny P and Leger R, Polymer 17:4594-4603 (2013). 